\title{
Decreased deformability of lymphocytes in chronic lymphocytic leukemia
}

\section{SUBJECT AREAS:}

BIOPHYSICS

BIOMEDICAL ENGINEERING

Received

20 August 2014

Accepted

3 December 2014

Published

9 January 2015

\section{Correspondence and} requests for materials should be addressed to

Y.S. (sun@mie. utoronto.ca) or C.W. (cwang@mtsinai.on.

Yi Zheng' ${ }^{1,2}$, Jun Wen', John Nguyen', Mark A. Cachia' ${ }^{1}$, Chen Wang ${ }^{4,5} \&$ Yu Sun ${ }^{1,2,3}$

'Department of Mechanical and Industrial Engineering, University of Toronto, Toronto, ON, Canada, ${ }^{2}$ Institute of Biomaterials and Biomedical Engineering, University of Toronto, Toronto, ON, Canada, ${ }^{3}$ Department of Electrical and Computer Engineering, University of Toronto, Toronto, ON, Canada, ${ }^{4}$ Department of Pathology and Laboratory Medicine, Mount Sinai Hospital, Toronto, ON, Canada, ${ }^{5}$ Department of Laboratory Medicine and Pathobiology, University of Toronto, Toronto, ON, Canada.

This paper reports the first study of stiffness/deformability changes of lymphocytes in chronic lymphocytic leukemia (CLL) patients, demonstrating that at the single cell level, leukemic metastasis progresses are accompanied by biophysical property alterations. A microfluidic device was utilized to electrically measure cell volume and transit time of single lymphocytes from healthy and CLL patients. The results from testing thousands of cells reveal that lymphocytes from CLL patients have higher stiffness (i.e., lower deformability), as compared to lymphocytes in healthy samples, which was also confirmed by AFM indentation tests. This observation is in sharp contrast to the known knowledge on other types of metastatic cells (e.g., breast and lung cancer cells) whose stiffness becomes lower as metastasis progresses.

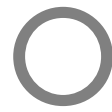

ver the past decade, there is growing evidence showing that cells undergo biophysical property changes during disease progression ${ }^{1,2}$. For example, the increased deformability of malignant cancer cells, as compared to benign cells, facilitates their migration and invasion ${ }^{3,4}$; and in sickle cell disease, sickled red blood cells due to their higher stiffness can cause vaso-occlusion ${ }^{5}$. Biophysical property changes of various leucocytes under a number of pathological conditions have been reported. Neutrophils were found to be significantly stiffened by formylmethionyl-leucyl-phenylalanine (FMLP) activation, and thereby took longer time to pass through $5 \mu \mathrm{m}$ capillary pores ${ }^{6}$. Neutrophils' deformability can be severely impaired in sepsis patients, and thus migration capability was consequently jeopardized ${ }^{7,8}$. Research has also shown that immature granulocytes and lymphocytes' inability to traverse micropipettes due to their compromised deformability, which can cause leukocclusive events, abnormal intravascular leukocyte aggregation and clumping in vasculature ${ }^{9}$. A recent micropipette aspiration study of lymphocytes from diabetic mice revealed that diabetic lymphocytes are stiffer than control cells, which is associated with several clinical complications ${ }^{10}$.

Leucocytes are nonadherent cells and can be tested with atomic force microscopy (AFM) when mechanically immobilized in microfabricated wells ${ }^{11,12}$. Using this technology, the stiffness alteration of acute lymphoblastic leukemia (ALL) cell lines (HL-60 and Jurkat) caused by chemotherapy was quantified ${ }^{13}$. The study concluded that after exposure to chemotherapy, leukemia cells possess increased stiffness, and the type of chemotherapy can affect stiffness kinetics. The increased cell stiffness in ALL patients with leucostasis symptoms was also shown by using the AFM technique ${ }^{12}$. Most recently, data obtained using optical tweezers provided preliminary evidence that leukemic hematopoietic cell populations in normal and leukemia patients with distinct primitiveness exhibited differential deformability ${ }^{14}$.

To understand the pathophysiology involving biophysical properties of cancerous leukocytes (leukemia), existing studies either performed measurement on cell lines instead of patient samples or tested a very limited number of cells. This paper presents the first study of stiffness/deformability changes of lymphocytes in chronic lymphocytic leukemia (CLL) patients based on the measurement of more than 1,000 cells per patient sample. The results reveal that lymphocytes from CLL patients have a higher stiffness (i.e., lower deformability), as compared to their counterparts in healthy samples, which is different from the known knowledge on other types of metastatic cells (e.g., breast and lung cancer cells) whose stiffness becomes lower as metastasis progresses.

\section{Methods}

Human lymphocytes mainly consist of B cells and T cells. Although previous studies have shown that CLL primarily affects B lymphocytes ${ }^{15}$, the exact origin of CLL disease is still controversial. In this study, the entire lymphocyte population was studied as a single group. Peripheral blood samples were collected from patients diagnosed with CLL and treated with ethylenediaminetetraacetic acid (EDTA, 1.5 mg/ml) as anticoagulant. Tests were performed in accordance with a research protocol approved by the Mount Sinai Hospital Review Board, and 
informed consent was obtained from all subjects. Mononuclear cells were isolated using a standard density-gradient protocol (Ficoll-Paque PLUS, GE Healthcare Bioscience, AB). Briefly, $4 \mathrm{ml}$ of diluted whole blood ( $2 \mathrm{ml}$ blood: $2 \mathrm{ml}$ PBS) was carefully layered on $3 \mathrm{ml}$ Ficoll-Paque PLUS, and then centrifuged at $400 \mathrm{~g}$ for $30 \mathrm{mins}$, which leaves the lymphocyte layer at the interface. The lymphocyte layer was resuspended in $6 \mathrm{ml} \mathrm{PBS}$ and centrifuged at $80 \mathrm{~g}$ for $10 \mathrm{~min}$ to remove platelets. After removing the supernatant, the cell pellet $(<5 \%$ monocytes and erythrocytes, $>95 \%$ lymphocytes) was re-suspended in PBS with $1 \% \mathrm{w} / \mathrm{v}$ BSA. A final density of $3 \mathrm{M} / \mathrm{ml}$ was used to significantly reduce the occurrence of multiple cells present in the measurement area and also to maintain a relatively high testing throughput. Samples were kept at room temperature for $30 \mathrm{~min}$ to reduce adhesion to channel walls. The testing of each sample was completed within 15 minutes at room temperature. To minimize potential artifacts during the preparation procedure, all samples were processed by strictly following this protocol.

Microfluidic devices were fabricated via standard PDMS soft lithography. The two stages of the microchannel [Fig. 1(a)] have cross-sectional areas of $5 \mu \mathrm{m} \times 5 \mu \mathrm{m}$ and $8.5 \mu \mathrm{m} \times 8.5 \mu \mathrm{m}$, respectively ${ }^{16}$. Two $\mathrm{Ag} / \mathrm{AgCl}$ electrodes $(200 \mu \mathrm{m}$ diameter $)$ connected to a lock-in amplifier (SI Fig. 1) were used to measure resistance changes at a sampling rate of $115 \mathrm{kHz}$ (time resolution $\sim 8.6 \mu \mathrm{s}$ ). Due to the blockage of electrical current, a single cell generates a pair of resistance peaks [Fig. 1(b)(c)] when driven through the channel under a negative pressure $(3 \mathrm{kPa})$. In Coulter counter, when a dielectric particle suspended in electrolytes transits through a small orifice, it produces a change of resistance that is proportional to the particle's volume $e^{17,18}$. The small peak and the large peak in our resistance profile correspond to the resistance increase when a cell passes through the $8.5 \mu \mathrm{m} \times 8.5 \mu \mathrm{m}$ channel and the $5 \mu \mathrm{m} \times$ $5 \mu \mathrm{m}$ channel, respectively. A threshold value was set to be $2 \%$ higher than the resistance without cells passing. The height of the smaller peak $(\Delta \mathrm{R})$ was used to measure cell volume according to the Coulter counter principle. The width of the larger peak (time span between the intercepts of the threshold and the peak profile) was used to determine transit time, $\Delta \mathrm{t}^{19,20}$. The events that multiple cells appeared simultaneously in the channel were excluded in post data processing.

The channel dimension of $8.5 \mu \mathrm{m} \times 8.5 \mu \mathrm{m}$ was specifically chosen to allow all lymphocytes to pass without being compressed by channel walls, and in the meanwhile, to achieve distinct volume measurement. The channel dimension of $5 \mu \mathrm{m} \times$ $5 \mu \mathrm{m}$ was selected to balance the need of constriction (smaller than the diameter of lymphocytes) and experimental practicality (e.g., ease of fabrication and reduction of clogging). The dead volume in between was designed to separate the two peaks in the resistance profile for the convenience of data processing. The length of both measurement units was chosen to be $15 \mu \mathrm{m}$ to lower the overall resistance of the system and achieve clear signal profiles when a cell passes through the measurement units.

Fig. 1(b) shows experimental data measured within $1 \mathrm{sec}(11 \mathrm{cells})$. Fig. 1(c) is the zoomed-in view of the circled area in Fig. 1(b). To extract the actual cell volume, finite element modeling was conducted in COMSOL. Cells possess complex electrical properties consisting of both capacitive and resistive components. However, in low frequency measurements, cells behave similarly to a dielectric object ${ }^{18,21}$. Thus, in finite element modeling, dielectric spheres with known sizes (diameter ranging from $5.0 \mu \mathrm{m}$ to $8.5 \mathrm{um}$ ) were simulated and corresponding electrical resistance values were determined. The relationship between sphere size and resistance change is shown in SI Fig. 2. Cells with a diameter larger than $8.5 \mu \mathrm{m}$ or smaller than 5 um were monocytes and red blood cells, and hence excluded in data processing.

\section{Results and Discussion}

Fig. 2(a) shows scatter plots of transit time vs. cell volume for lymphocytes from a control/healthy sample $(\mathrm{n}=1,048)$ and a CLL sample $(n=796)$. The distributions of cell volume and transit time are presented in Fig. 2(b). Transit time is mainly affected by cell volume and cell deformability. We first investigated whether cell volume alone can explain the transit time variation. Within the same sample, lymphocytes with larger volume indeed took longer time to travel through the microfluidic channel. Our measured transit time exhibited a power law dependence on cell volume in agreement with previous studies where cell is modeled as a viscous liquid drop ${ }^{22,23}$. It is worth noting that within the same sample, a $2 \mu \mathrm{m}$ change in cell diameter caused transit time to span more than an order of magnitude, which indicates the dominant effect of cell size/volume on transit time.

However, with the same diameter, transit time of the lymphocytes varies by 3-4 folds, which indicates the effect of cell deformability differences. As shown in Fig. 2(a), the median cell diameter and transit time of the control sample were measured to be $7.20 \mu \mathrm{m}$ and $4.81 \mathrm{~ms}$; whereas the median cell diameter and transit time are $6.92 \mu \mathrm{m}$ and $5.63 \mathrm{~ms}$ for the CLL sample. Cells in the CLL sample are smaller compared to cells in the control sample but take longer time to pass through the $5 \mu \mathrm{m} \times 5 \mu \mathrm{m}$ channel. This fact led us to hypothesize that lymphocytes in CLL patients have decreased deformability.

Cell volume and transit time from 5 control samples and 5 CLL samples were measured using the microfluidic system. As shown in Fig. 3(a)(b), for each sample, the three lines of the box represent 75
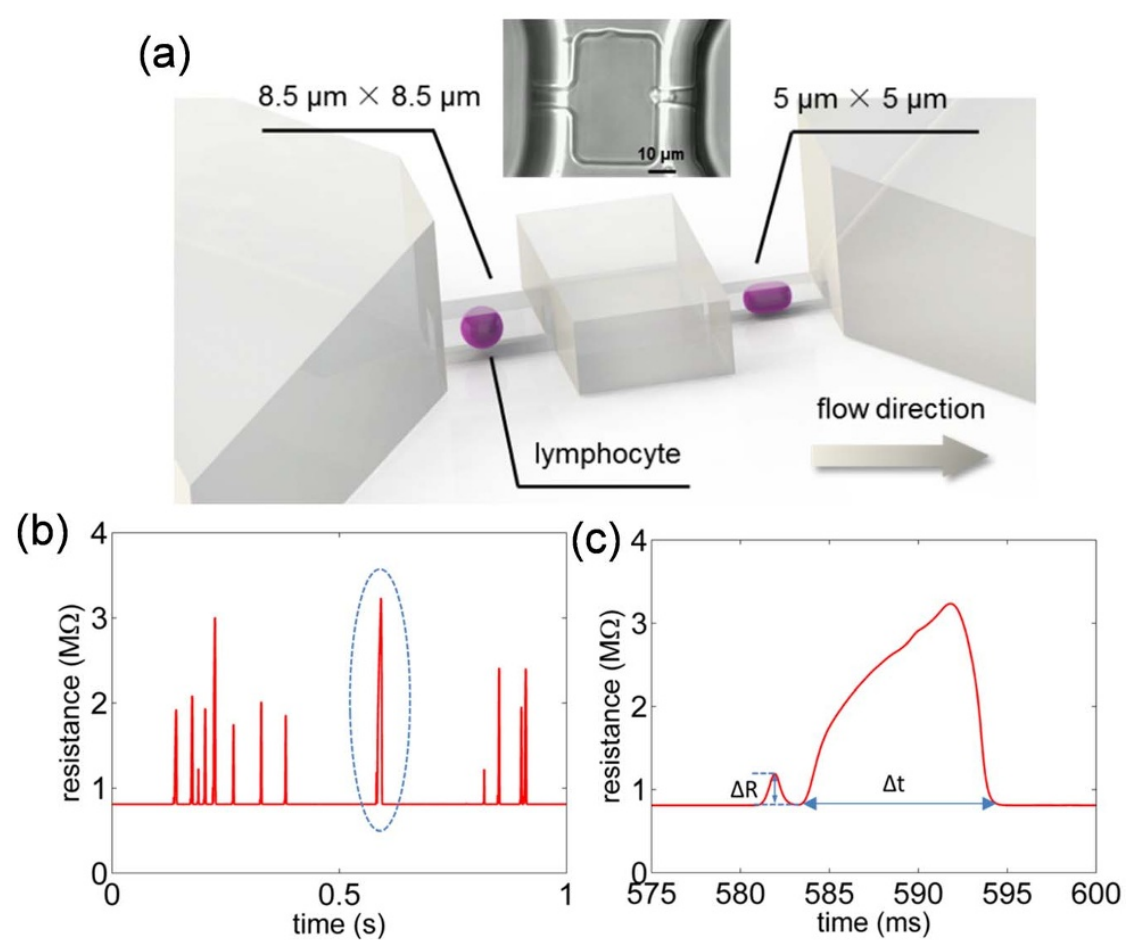

Figure 1 (a) Microfluidic system for electrically measuring lymphocytes volume and transit time. When a lymphocyte is driven through the measurement units under a negative pressure $(3 \mathrm{kPa})$ (also see SI Video), the total electrical resistance of the channel increases due to the blockage of electrical current. (b) Experimental resistance data recorded within 1 second. Zoom-in of the circled area is shown in (c). Cell volume and transit time are determined by measuring $\Delta \mathrm{R}$ and $\Delta \mathrm{t}$. 

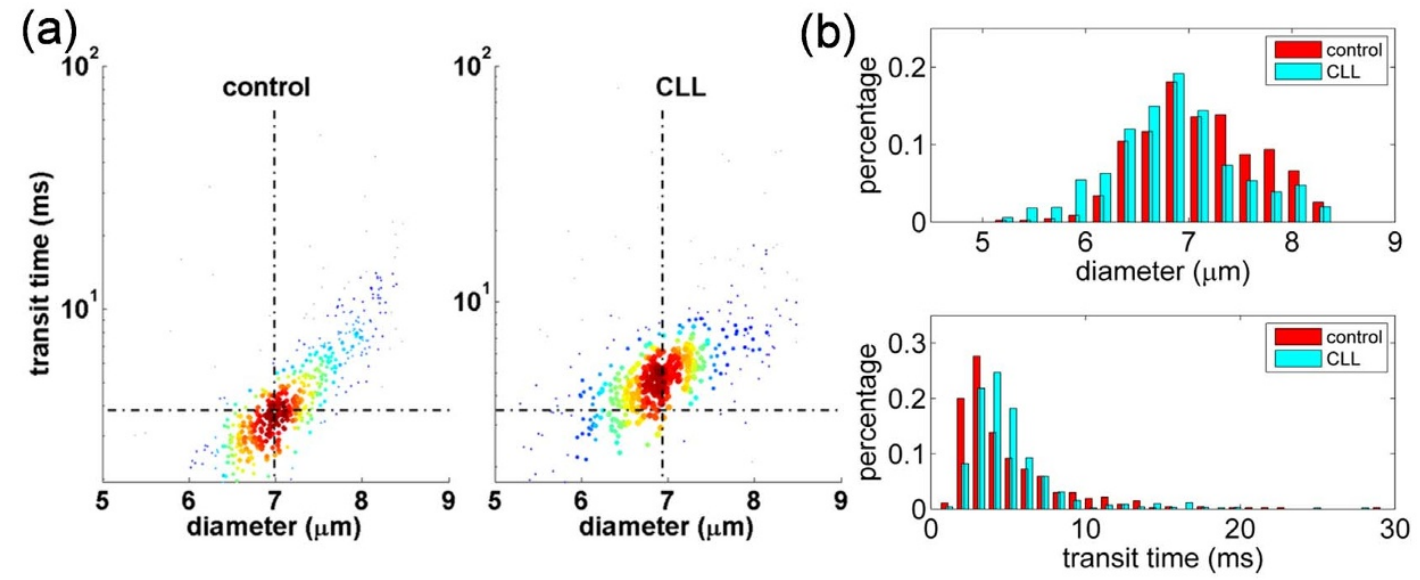

Figure $2 \mid$ (a) Scatter plots of transit time vs. cell diameter for lymphocytes from a control/healthy sample $(\mathrm{n}=1,048)$ and a CLL sample $(\mathrm{n}=796)$. Transit time exhibits a power law dependence on cell volume/diameter. (b) Histograms of cell diameter and transit time of the control and CLL samples.

percentile, median, and 25 percentile. The whiskers represent the locations of maximum and minimum. Fig. 3(c)(d) summarize average group median values of transit time and cell volume with error bars representing standard error of the mean. Compared to control samples, CLL samples have a smaller cell volume $(7.1 \pm 0.06 \mu \mathrm{m}$ vs. $7.3 \pm 0.03 \mu \mathrm{m})$ but a higher median transit time $(5.1 \pm 0.44 \mathrm{~ms}$ vs. $3.5 \pm 0.16 \mathrm{~ms}$ ), indicating that CLL lymphocytes are less deformable. The volume measurement results fall in the same range as reported in previous studies ${ }^{24}$. Statistical difference exists between the two populations.

Furthermore, as shown in Fig. 3(a), 75 percentile, median, and 25 percentile cell transit time values were all higher for CLL samples, demonstrating that the measured transit time difference reflects entire cell populations tested in this work. Fig. 3 (e)(f) present the difference of 75 and 25 percentile of cell transit time and diameter. Cell transit time variance is more prominent in CLL samples, likely attributed to the increased heterogeneity caused by accumulated mutations during malignancies ${ }^{25}$. SI Fig. 3 presents the relationship of median transit time and median diameter of the control and CLL samples. There are samples with slightly smaller volume exhibiting longer transit time (for both control and CLL samples), indicating that deformability variation in addition to cell volume also contributes to the observed transit time difference. Note that since none of the CLL patients showed leukostasis symptoms, all the lymphocytes were able to pass through the microchannel ${ }^{26}$.

It has been reported that changing the coating of channel surfaces from PEG to PLL can cause cell entry velocity and transit velocity to decrease $^{22}$, indicating that surface properties can possibly contribute to transit time differences by influencing the friction between cell membrane and microfluidic channel walls. In our work, the isolated lymphocytes were incubated in PBS with $1 \%$ BSA for $30 \mathrm{~min}$ to reduce friction ${ }^{16}$. Before loading cells, the microfluidic channel was also perfused with PBS $+1 \%$ BSA for 30 min to further reduce friction force of control and CLL lymphocytes. To confirm the measured transit time difference (control vs. CLL) truly reflects cell deformability/stiffness, lymphocytes from a control sample and a CLL sample were tested using AFM indentation.

Lymphocytes' stiffness was measured when the cells were immersed in PBS at room temperature using an AFM (Bioscope Catalyst, Santa Barbara, CA) mounted on an inverted microscope. The cells were mechanically immobilized within microwells ${ }^{11,12}$. AFM indentation was conducted in contact mode using a triangular silicon nitride cantilever with a nominal spring constant of $0.03 \mathrm{~N} / \mathrm{m}$ (MSCT-D, Veeco Probes, Camarillo, CA). The spring constant was calibrated using the thermal noise method in the software
(Nanoscope 8.10) provided by the AFM manufacturer. Forceindentation curves were collected with a loading speed of $20 \mu \mathrm{m} / \mathrm{s}$, and a triggering force of $500 \mathrm{pN}$ was set to minimize substrate effect.

(a) (c)

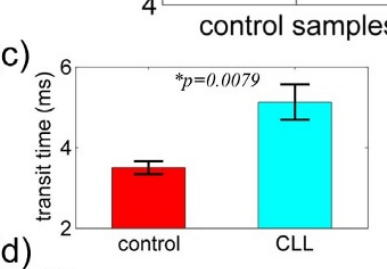

d)

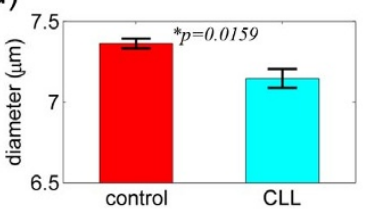

(b)
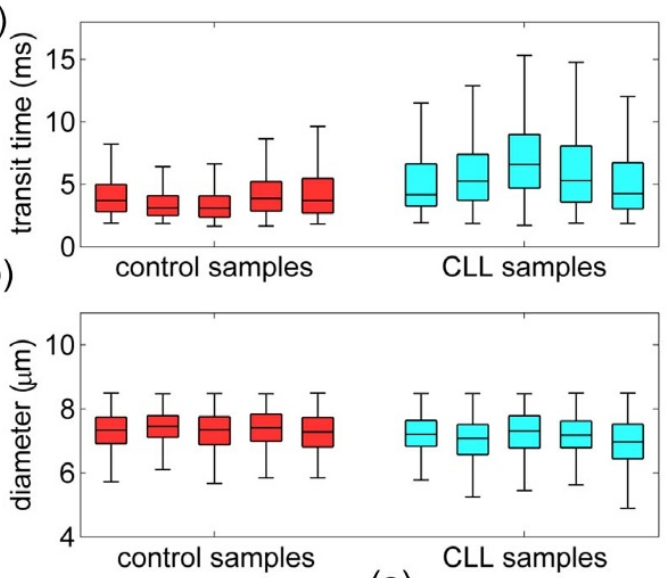

(e)

(f)
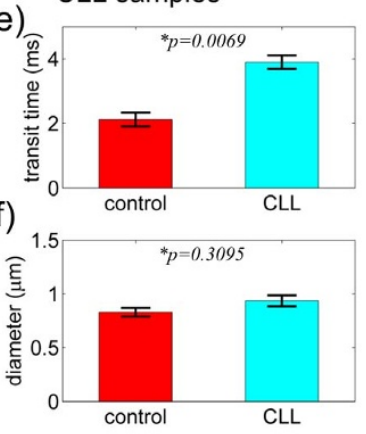

Figure 3 $\mid$ Transit time (a) and cell volume (b) measured from 5 control/ healthy samples (red) and 5 CLL samples (cyan). For each sample, the three lines of the box represent 75 percentile, median, and 25 percentile; the whiskers represent the locations of maximum and minimum $(n \approx 1,000$ for each sample). The average median values of transit time (c) and cell diameter (d) of the 5 control samples and the 5 CLL samples are $3.5 \pm$ $0.16 \mathrm{~ms}$ vs. $5.1 \pm 0.44 \mathrm{~ms}$ and $7.3 \pm 0.03 \mu \mathrm{m}$ vs. $7.1 \pm 0.06 \mu \mathrm{m}$, respectively. In general, cells in CLL samples are slightly smaller than cells in control samples but reveal a longer transit time, indicating that they are less deformable. (e) and (f) summarize the difference of 75 and 25 percentile of cell transit time and diameter. * $p$ was calculated using MannWhitney nonparametric analysis; and error bars represent standard error of the mean value. 
(a)

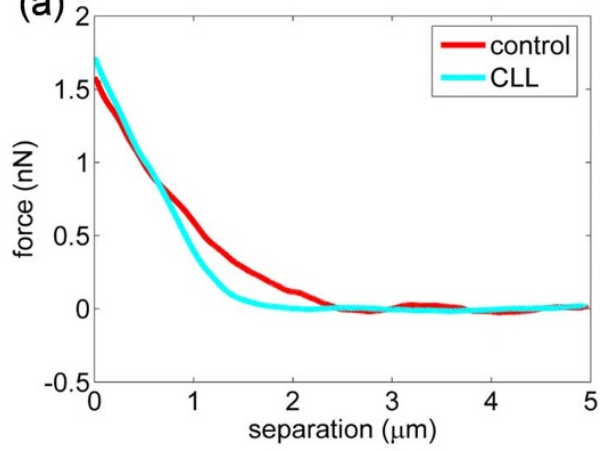

(b)

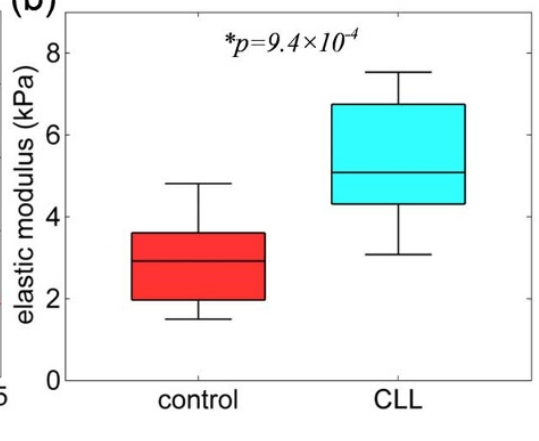

Figure $4 \mid$ (a) Experimental AFM indentation curves of two representative lymphocytes, one from control sample and one from CLL sample. The elastic moduli of lymphocytes were quantitatively determined by fitting force-indentation curves to standard Hertz model for a pyramidal tip. (b) Elastic modulus values of the control sample $(\mathrm{n}=10)$ and the CLL sample $(\mathrm{n}=14) .{ }^{*} p$ was calculated using Mann-Whitney nonparametric analysis.

Baseline corrections were applied to the raw curves to eliminate curve inclination before tip-cell contact. The elastic moduli of lymphocytes were quantitatively determined by fitting the forceindentation curves (contact zone) to the standard Hertz model for pyramidal tips. The half angle and the radius of the pyramidal AFM tip were set to be $14^{\circ}$ and $10 \mathrm{~nm}$, respectively in calculation. In all Hertz model fitting, $\mathrm{R}^{2}$ is above 0.99. Fig. 4(a) shows experimental indentation curves of two representative lymphocytes from a control sample $(n=10)$ and a CLL sample $(n=14)$. The elastic moduli of control and CLL samples are summarized in Fig. 4(b) (mean \pm SEM: $2.9 \pm 0.36 \mathrm{kPa}$ and $5.4 \pm 0.38 \mathrm{kPa}$ ). This difference in elastic modulus confirms that lymphocytes in CLL patients have a higher stiffness than lymphocytes in control samples, and the higher stiffness contributes to the longer transit time of CLL lymphocytes, although a quantitative correlation between AFM results with cell transit time from microfluidic measurement cannot be established.

In AFM indentation, the choice of cells to test was arbitrary. The use of microwells to confine cells could also increase cell tension; therefore, relatively loose confinement is desired. Additionally, the heterogeneity of cell structures and local measurement of AFM can also induce variance in the quantified elastic modulus values (In this study, the average value of three indentations on different locations was used as the cell's elastic modulus). In comparison, the microfluidic testing is relatively easier to control and can better reveal the inter-sample variability. Thus, the AFM results are only used to provide qualitative evidence that CLL lymphocytes are mechanically stiffer.

Difference in actin filament density was often thought responsible for deformability differences between two cell types ${ }^{19,22}$. However, a decrease in actin content of lymphocytes from CLL patients was previously found ${ }^{27}$. In addition, no connection was found between actin level and cell deformability in human leukemia cell lines ${ }^{11}$. Recently, vimentin (intermediate filaments) was shown to have dominant influence on mouse lymphocyte deforma$\operatorname{tion}^{28}$. In our study, we noticed that the nuclei of CLL lymphocytes occupy almost the entire cell [SI Fig. 4]. As a result, when they flow through the microfluidic channel, the nuclei of CLL lymphocytes could have contributed more significantly to transit time since cell nuclei are known to be significantly stiffer compared to cytoplasm and cell membrane $e^{9,29,30}$. Thus, the enlarged nuclei may be a factor that contributes to the longer transit time observed for CLL lymphocytes. In the AFM measurements with an indentation depth of $2 \mu \mathrm{m}$, the nuclear effect should have reflected itself in the quantified elastic modulus. However, the exact pathological causes and the underlying mechanism of the observed deformability difference in lymphocytes from healthy and CLL patients necessitate further investigation.

\section{Conclusion}

This paper reports stiffness/deformability differences of lymphocytes from healthy donors and chronic lymphocytic leukemia (CLL) patients. Microfluidic measurement was used to quantify cell volume and transit time of thousands of lymphocytes from control and CLL samples. The results reveal that CLL lymphocytes have higher stiffness (i.e., lower deformability), as compared to lymphocytes in healthy samples. Their higher stiffness was also confirmed via AFM indentation. This study demonstrates that at the single cell level, leukemic metastasis progresses are accompanied by biophysical property alterations. Further work involving a large sample size could reveal the heterogeneity of CLL lymphocyte deformability and establish a correlation of deformability alterations and clinical symptoms. Technically, the development of biophysical models can enable the extraction of lymphocyte elastic modulus from the measured cell volume and transit time.

1. Plodinec, M. et al. The nanomechanical signature of breast cancer. Nat. Nanotechnol. 7, 757-765 (2012).

2. Zheng, Y., Nguyen, J., Wei, Y. \& Sun, Y. Recent advances in microfluidic techniques for single-cell biophysical characterization. Lab Chip 13, 2464-2483 (2013).

3. Gossett, D. R. et al. Hydrodynamic stretching of single cells for large population mechanical phenotyping. Proc. Natl. Acad. Sci. U. S. A. 109, 7630-7635 (2012).

4. Shojaei-Baghini, E., Zheng, Y., Jewett, M. A. S., Geddie, W. B. \& Sun, Y. Mechanical characterization of benign and malignant urothelial cells from voided urine. Appl. Phys. Lett. 102, 123704-123704 (2013).

5. Wood, D. K., Soriano, A., Mahadevan, L., Higgins, J. M. \& Bhatia, S. N. A Biophysical Indicator of Vaso-occlusive Risk in Sickle Cell Disease. Sci. Transl. Med. 4, 123ra26 (2012)

6. Frank, R. S. Time-Dependent Alterations in the Deformability of Human Neutrophils in Response to Chemotactic Activation. Blood 76, 2606-2612 (1990).

7. Kovach, M. A. \& Standiford, T. J. The function of neutrophils in sepsis. Curr. Opin. Infect. Dis. 25, 321-327 (2012).

8. Skoutelis, A. T. et al. Neutrophil deformability in patients with sepsis, septic shock, and adult respiratory distress syndrome. Crit. Care Med. 28, 2355-2359 (2000).

9. Lichtman, M. A. Rheology of Leukocytes, Leukocyte Suspensions, and Blood in Leukemia - Possible Relationship to Clinical Manifestations. J. Clin. Invest. 52, 350-358 (1973).

10. Perrault, C. M., Bray, E. J., Didier, N., Ozaki, C. K. \& Tran-Son-Tay, R. Altered rheology of lymphocytes in the diabetic mouse. Diabetologia 47, 1722-1726 (2004).

11. Rosenbluth, M. J., Lam, W. A. \& Fletcher, D. A. Force microscopy of nonadherent cells: A comparison of leukemia cell deformability. Biophys. J. 90, 2994-3003 (2006).

12. Lam, W. A., Rosenbluth, M. J. \& Fletcher, D. A. Increased leukaemia cell stiffness is associated with symptoms of leucostasis in paediatric acute lymphoblastic leukaemia. Br. J. Haematol. 142, 497-501 (2008).

13. Lam, W. A., Rosenbluth, M. J. \& Fletcher, D. A. Chemotherapy exposure increases leukemia cell stiffness. Blood 109, 3505-3508 (2007).

14. Tan, Y. H. et al. Biophysical characterization of hematopoietic cells from normal and leukemic sources with distinct primitiveness. Appl. Phys. Lett. 99 (2011).

15. Chiorazzi, N., Rai, K. R. \& Ferrarini, M. Chronic lymphocytic leukemia. N. Engl. J. Med. 352, 804-815+850 (2005). 
16. Zheng, Y., Nguyen, J., Wang, C. \& Sun, Y. Electrical measurement of red blood cell deformability on a microfluidic device. Lab Chip 13, 3275-3283 (2013).

17. Deblois, R. W. \& Bean, C. P. Counting and Sizing of Submicron Particles by Resistive Pulse Technique. Rev. Sci. Instrum. 41, 909-\& (1970).

18. Sun, T. \& Morgan, H. Single-cell microfluidic impedance cytometry: a review. Microfluid Nanofluid 8, 423-443 (2010).

19. Gabriele, S., Benoliel, A. M., Bongrand, P. \& Theodoly, O. Microfluidic Investigation Reveals Distinct Roles for Actin Cytoskeleton and Myosin II Activity in Capillary Leukocyte Trafficking. Biophys. J. 96, 4308-4318 (2009).

20. Zheng, Y., Shojaei-Baghini, E., Azad, A., Wang, C. \& Sun, Y. High-throughput biophysical measurement of human red blood cells. Lab Chip 12, 2560-2567 (2012)

21. Zheng, Y., Shojaei-Baghini, E., Wang, C. \& Sun, Y. Microfluidic characterization of specific membrane capacitance and cytoplasm conductivity of single cells. Biosens. Bioelectron. 42, 496-502 (2013).

22. Byun, S. et al. Characterizing deformability and surface friction of cancer cells. Proc. Natl. Acad. Sci. U. S. A. 110, 7580-7585 (2013).

23. Tsai, M. A., Frank, R. S. \& Waugh, R. E. Passive Mechanical-Behavior of Human Neutrophils - Power-Law Fluid. Biophys. J. 65, 2078-2088 (1993).

24. Kuse, R., Schuster, S., Schubbe, H., Dix, S. \& Hausmann, K. Blood Lymphocyte Volumes and Diameters in Patients with Chronic Lymphocytic-Leukemia and Normal Controls. Blut 50, 243-248 (1985).

25. Schuh, A. et al. Monitoring chronic lymphocytic leukemia progression by whole genome sequencing reveals heterogeneous clonal evolution patterns. Blood 120, 4191-4196 (2012).

26. Rosenbluth, M. J., Lam, W. A. \& Fletcher, D. A. Analyzing cell mechanics in hematologic diseases with microfluidic biophysical flow cytometry. Lab Chip $\mathbf{8}$, 1062-1070 (2008).

27. Stark, R., Liebes, L. F., Nevrla, D., Conklyn, M. \& Silber, R. Decreased Actin Content of Lymphocytes from Patients with Chronic Lymphocytic-Leukemia. Blood 59, 536-541 (1982).

28. Brown, M. J., Hallam, J. A., Colucci-Guyon, E. \& Shaw, S. Rigidity of circulating lymphocytes is primarily conferred by vimentin intermediate filaments. J. Immunol. 166, 6640-6646 (2001).

29. Pajerowski, J. D., Dahl, K. N., Zhong, F. L., Sammak, P. J. \& Discher, D. E. Physical plasticity of the nucleus in stem cell differentiation. Proc. Natl. Acad. Sci. U. S. A. 104, 15619-15624 (2007).
30. Liu, H. et al. In Situ Mechanical Characterization of the Cell Nucleus by Atomic Force Microscopy. ACS Nano 8, 3821-3828 (2014).

\section{Acknowledgments}

Financial support from the Natural Sciences and Engineering Research Council of Canada (NSERC) through an E. W. R. Steacie Fellowship, from Grand Challenges of Canada, and from the Canada Research Chairs Program is acknowledged.

\section{Author contributions}

Y.Z., Y.S. and C.W. designed the project. Y.Z. performed microfluidic experiemnts and processed data. J.W. performed AFM experiements. J.N. developed the signal processing algorithms. M.A.C. preprocessed blood samples and participated in microfluiic experiments. Y.Z., C.W. and Y.S. discussed resutls and constructed the manuscript. All authors reviewed the manuscript.

\section{Additional information}

Supplementary information accompanies this paper at http://www.nature.com/ scientificreports

Competing financial interests: The authors declare no competing financial interests. How to cite this article: Zheng, Y. et al. Decreased deformability of lymphocytes in chronic lymphocytic leukemia. Sci. Rep. 5, 7613; DOI:10.1038/srep07613 (2015).

This work is licensed under a Creative Commons Attribution-NonCommercialNoDerivs 4.0 International License. The images or other third party material in this article are included in the article's Creative Commons license, unless indicated otherwise in the credit line; if the material is not included under the Creative Commons license, users will need to obtain permission from the license holder in order to reproduce the material. To view a copy of this license, visit http:// creativecommons.org/licenses/by-nc-nd/4.0/ 